This is the author's copy of the publication as archived with the DLR's electronic library at http://elib.dlr.de. Please consult the original publication for citation, see e.g. https://ieeexplore.ieee.org/document/9172659

\title{
MMX - Development of a Rover Locomotion System for Phobos
}

Sedlmayr, Hans-Jürgen und Barthelmes, Stefan und Bayer, Ralph und Bertleff, Wieland und Bihler, Markus und Buse, Fabian und Chalon, Maxime und Franke, Denis und Ginner, Florian und Langofer, Viktor und Lichtenheldt, Roy und Obermeier, Thomas und Pignede, Antoine Francois Xavier und Reill, Josef und Skibbe, Juliane

MMX (Martian Moons eXploration) is a robotic sample return mission of JAXA (Japan Aerospace Exploration Agency), CNES (Centre National d' Études Spatiales), and DLR (German Aerospace Center) with the launch planned for 2024. The mission aims to answer the question of the origin of Phobos and Deimos, which will also help to understand the material transport in the earliest period of our solar system, and of how was water brought to Earth. Besides JAXA's MMX mothership, which is responsible for sampling and sample return to Earth, a small rover which is built by CNES and DLR to land on Phobos for in-situ measurements, similar to MASCOT (Mobile Asteroid Surface Scout) on Ryugu. The MMX rover is a fourwheel driven autonomous system with a size of $41 \mathrm{~cm}$ $\times 37 \mathrm{~cm} \times 30 \mathrm{~cm}$ and a weight of approximately $25 \mathrm{~kg}$. Multiple science instruments and cameras are integrated in the rover body. The rover body has the form of a rectangular box. Attached at the sides are four legs with one wheel per leg. When the rover is detached from the mothership, the legs are folded together at the side of the rover body. When the rover has landed passively (no parachute or braking rockets) on Phobos, the legs are autonomously maneuvered to bring the rover in an upright orientation. One Phobos day lasts 7.65 earth hours, which yields about 300 extreme temperature cycles for the total mission time of three earth months. These cycles and the wide span of surface temperature between day and night are the main design drivers for the rover. This paper gives a detailed view on the development of the MMX rover locomotion subsystem

\section{Copyright Notice}

(C2020 IEEE. Personal use of this material is permitted. Permission from IEEE must be obtained for all other uses, in any current or future media, including reprinting/republishing this material for advertising or promotional purposes, creating new collective works, for resale or redistribution to servers or lists, or reuse of any copyrighted component of this work in other works.

Sedlmayr, Hans-Jürgen und Barthelmes, Stefan und Bayer, Ralph und Bertleff, Wieland und Bihler, Markus und Buse, Fabian und Chalon, Maxime und Franke, Denis und Ginner, Florian und Langofer, Viktor und Lichtenheldt, Roy und Obermeier, Thomas und Pignede, Antoine Francois Xavier und Reill, Josef und Skibbe, Juliane (2020) MMX - development of a rover locomotion system for Phobos. In: 2020 IEEE Aerospace Conference, AERO 2020. Aerospace Conference, 07.03.2020 - 14.03.2020, Big Sky, Montana, USA. ISBN 978-172812734-7 ISSN 1095-323X 


\section{MMX - Development of a Rover Locomotion System for Phobos}

\author{
Hans-Juergen Sedlmayr \\ German Aerospace Center (DLR) \\ Muenchener Str. 20 \\ 82234 Wessling, Germany \\ Hans-Juergen.Sedlmayr@dlir.de \\ Wieland Bertleff \\ German Aerospace Center (DLR) \\ Muenchener Str. 20 \\ 82234 Wessling, Germany \\ Wieland.Bertleff@dlr.de \\ Maxime Chalon \\ German Aerospace Center (DLR) \\ Muenchener Str. 20 \\ 82234 Wessling, Germany \\ Maxime.Chalon@dlr.de \\ Viktor Langofer \\ German Aerospace Center (DLR) \\ Muenchener Str. 20 \\ 82234 Wessling, Germany \\ Viktor.Langofer@dlr.de \\ Antoine Pignède \\ German Aerospace Center (DLR) \\ Muenchener Str. 20 \\ 82234 Wessling, Germany \\ Antoine.Pignede@dlr.de
}

\author{
Stefan Barthelmes \\ German Aerospace Center (DLR) \\ Muenchener Str. 20 \\ 82234 Wessling, Germany \\ Stefan.Barthelmes@dlr.de \\ Markus.Bihler \\ German Aerospace Center (DLR) \\ Muenchener Str. 20 \\ 82234 Wessling, Germany \\ Markus.Bihler@dlr.de \\ Dennis Franke \\ German Aerospace Center (DLR) \\ Muenchener Str. 20 \\ 82234 Wessling, Germany \\ Dennis.Franke@dlr.de
}

Roy Lichtenheldt

German Aerospace Center (DLR)

Muenchener Str. 20

82234 Wessling, Germany

Roy.Lichtenheldt@dlr.de

Josef Reill

German Aerospace Center (DLR)

Muenchener Str. 20

82234 Wessling, Germany

Josef.Reill@dlr.de

Simon Tardivel

Centre National d'Etudes Spatiales (CNES)

18 avenue $E$. Belin

31401 Toulouse cedex 9, France

Simon.Tardivel@cnes.fr

\author{
Ralph Bayer \\ German Aerospace Center (DLR) \\ Muenchener Str. 20 \\ 82234 Wessling, Germany \\ Ralph.Bayer@dlr.de \\ Fabian Buse \\ German Aerospace Center (DLR) \\ Muenchener Str. 20 \\ 82234 Wessling, Germany \\ Fabian.Buse@dlr.de \\ Florian Ginner \\ German Aerospace Center (DLR) \\ Muenchener Str. 20 \\ 82234 Wessling, Germany \\ Florian.Ginner@dlr.de \\ Thomas Obermeier \\ German Aerospace Center (DLR) \\ Muenchener Str. 20 \\ 82234 Wessling, Germany \\ Thomas.Obermeier@dlr.de \\ Juliane Skibbe \\ German Aerospace Center (DLR) \\ Muenchener Str. 20 \\ 82234 Wessling, Germany \\ Juliane.Skibbe@dlr.de
}

\begin{abstract}
MMX (Martian Moons eXploration) is a robotic sample return mission of JAXA (Japan Aerospace Exploration Agency), CNES (Centre National d' Études Spatiales), and DLR (German Aerospace Center) with the launch planed for 2024. The mission aims to answer the question of the origin of Phobos and Deimos, which will also help to understand the material transport in the earliest period of our solar system, and of how was water brought to Earth. Besides JAXA's MMX mothership, which is responsible for sampling and sample return to Earth, a small rover which is built by CNES and DLR to land on Phobos for in-situ measurements, similar to MASCOT (Mobile Asteroid Surface Scout) on Ryugu. The MMX rover is a fourwheel driven autonomous system with a size of $41 \mathrm{~cm} \times 37 \mathrm{~cm}$ $x 30 \mathrm{~cm}$ and a weight of approximately $25 \mathrm{~kg}$. Multiple science instruments and cameras are integrated in the rover body. The rover body has the form of a rectangular box. Attached at the sides are four legs with one wheel per leg. When the rover is detached from the mothership, the legs are folded together at the side of the rover body. When the rover has landed passively (no parachute or braking rockets) on Phobos, the legs are autonomously maneuvered to bring the rover in an upright orientation. One Phobos day lasts $\mathbf{7 . 6 5}$ earth hours, which yields about 300 extreme temperature cycles for the total mission time of three earth months. These cycles and the wide span of surface temperature between day and night are the main design drivers for the rover. This paper gives a detailed view on the development of the MMX rover locomotion subsystem.
\end{abstract}

978-1-7281-2734-7/20/\$31.00 @2020 European Union
Table of Contents

1. INTRODUCTION - THE MMX MISSION ........ 1

2. THE ROVER ............................. 2

3. THE ROVER LOCOMOTION SUBSYSTEM........ 2

4. AnAlysis And testing $\ldots \ldots \ldots \ldots \ldots \ldots \ldots \ldots$. 7

5. Conclusion $\ldots \ldots \ldots \ldots \ldots \ldots \ldots \ldots \ldots \ldots \ldots, 8$

REFERENCES $\ldots \ldots \ldots \ldots \ldots \ldots \ldots \ldots \ldots \ldots \ldots, 8$

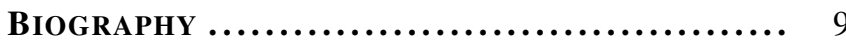

\section{INTRODUCTION - THE MMX MISSION}

After the success of the asteroid lander MASCOT from DLR and CNES (see [1] and [2]) to JAXA's Hayabusa2 mission in 2018 , a new target was put in place to expand this fruitful collaboration of the three space agencies to future missions. One of the next milestones in JAXA's roadmap for space exploration is the investigation of the Martian moon Phobos. For this Martian Moons Exploration (MMX) mission [3], DLR and CNES were invited to contribute a lander that is able to move on Phobos's surface. The requirements of the lander are to de-risk the landing of the main spacecraft and to contribute to the scientific objectives of MMX with its own scientific instruments. As Phobos gravity is in the range of $0.3-0.7 \mathrm{mg}$, it is deemed be feasible to use hopping principle similar to the mechanism that was used in MASCOT on 


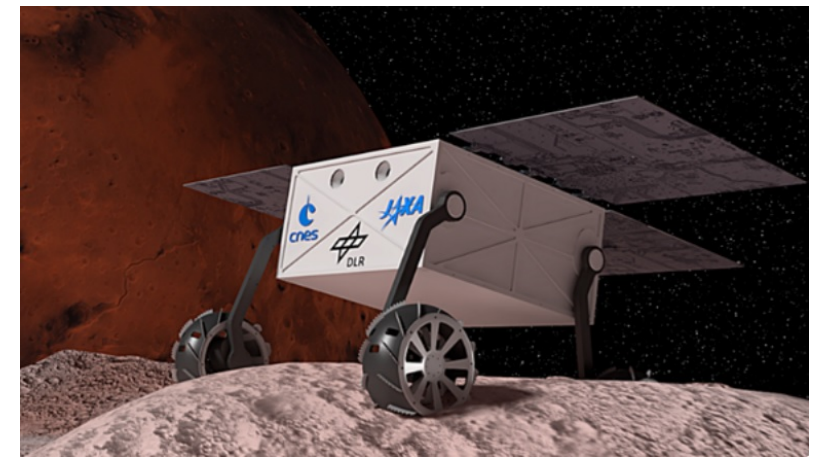

Figure 1. Rendering of the first MMX rover concept (credits by $C N E S)$

asteroid Ryugu. First analysis showed that a wheeled rover would also be able to drive at speeds of up to $20 \mathrm{~mm} / \mathrm{sec}$ considering rough terrain and low gravity. With a mission duration of several weeks, this seems to be a sufficient speed to reach different points of interest safely. One advantage of the wheeled locomotion concept is to use the wheel imprint on the Phobos soil to investigate the regolith behaviour on Phobos. The first rover concept is shown in Figure 1.

The orbiter, acting as a transponder between the rover and the Earth, will not stay in visibility for more than a couple of hours per Phobos day. As a result, the lander needs to be able to operate semi-autonomously. The small rover would benefit from being able to navigate and perform hazard avoidance tasks on its own. Even the uprighting phase right after the ballistic landing will require autonomy as a communication link will not be available during this phase. With the given scenario it was decided to use a roving unit with stereo based cameras for perception to navigate and carry scientific payload safely. The actual concept considers four legs equipped with wheels and skid-steering to meet mass constraint requirements. The legs become necessary as the rovers's orientation after landing is not defined and the system needs to be able to upright itself into an operable position. The environmental conditions of Phobos are roughly known from precursor missions such as Mars Express, Mars Global Surveyor or Mars Reconnaissance Orbiter. Mars Express was initially focused on the investigation of Mars's surface, interior and atmosphere but was also able to perform Phobos flybys at a distance of less than $100 \mathrm{~km}$. Images at resolutions of about 1.5 meters per pixel are available in some areas.

Given the size and gravity of Phobos, the density is estimated at around $1.85 \mathrm{~g} / \mathrm{cm}^{3} \pm 0.07$. Surface temperatures of $-112^{\circ} \mathrm{C}$ to $+25^{\circ} \mathrm{C}$ are to be expected. This, together with tight mass and energy budgets, pose a great challenge to developing a reliable mechatronic system for the mission. Figure 1 shows a first draft of the rover system with the body envelope size of $41 \times 37 \times 30 \mathrm{~cm}$. The overall mass is fixed to about $25 \mathrm{~kg}$ with scientific payload mass of $2 \mathrm{~kg}$.

The rest of the paper is organized as the following: Section 2 introduces the overall design concept of the MMX rover; Section 3 brings the focus to the locomotion subsystem of the rover at a more detailed level; Section 4 for describes the testing and analysis methods for validation of the rover design; finally, concluding remarks and outlook for the mission is given in Section 5.

\section{THE ROVER}

The rover consists of a body with a warm and cold compartment, four legs with one wheel each, one patch antenna on the body and three solar cell arrays which are unstowed after landing and uprighting. The cold environmental conditions of Phobos are the design driver to concentrate all electronics inside of the rover body. Even the motors are located in the rover body and the rotational movement is transmitted to the wheels by the usage of a crown gear and a pole in the rover leg. This way the wheels do not need heaters and the energy is saved for actuation and computation power. Due to thermal considerations the stereo cameras are also located in the rover body as a camera mast would need additional heaters.

The current rover design can accommodate four principal investigator instruments:

- NavCAM - cameras for autonomous navigation and imaging science, CMOS 2048 x 2048 with a RGB Bayer filter

- WheelCAM - two identical cameras, CMOS 2048 x 2048 , observing the wheel interaction on the ground, with a spatial resolution of under $100 \mu \mathrm{m}$ per pixel

- RAX - Raman Spectrometer to characterize the mineralogy of soil under the rover (developed by DLR, INTA/UVA and JAXA), with heritage from the ExoMars Raman spectrometer - miniRAD - for measurements of thermo-physical parameters with heritage from MASCOT [2] radiometer MARA

As the rover is a joint project of DLR and CNES, the contribution is divided evenly, and notably, for the main systems:

- power system (CNES)

- RF-system (CNES)

- OBC (CNES)

- thermal engineering (CNES)

- flight software, CCDH (CNES)

- cameras (CNES)

- chassis (DLR)

- shutters (DLR)

- harness (DLR)

- MECSS - mechanical electrical support system (DLR)

- electronics box (DLR)

- locomotion subsystem (DLR)

For more information on the rover mission, development, constraints, and design, please refer to Tardivel, Lange et al., 2019 [4]. For more information on the science of the rover, please refer to the Ulamec et al., 2019 [5]. For more information on the robotics challenges the rover faces on Phobos, please refer to Bertrand et al., 2019 [6].

\section{THE ROVER LOCOMOTION SUBSYSTEM}

\section{Electronic components}

As Phobos requires the rover to survive demanding thermal conditions, the main body consists of an inner compartment and an outer box. For the purpose of thermal control the inner part has around itself a thermal insulation and the outer box on the inner side of its panels. As space and weight is limited in the rover, the locomotion subsystem has no redundancy on the in neither the electrical nor mechanical part.

The electronics design allows all wheels and legs to be moved individually and simultaneously. For the purpose of reliable and low power communication with the onboard computer (OBC) a standard spacewire link is used. The electronics architecture of the locomotion subsystem and its main inter- 


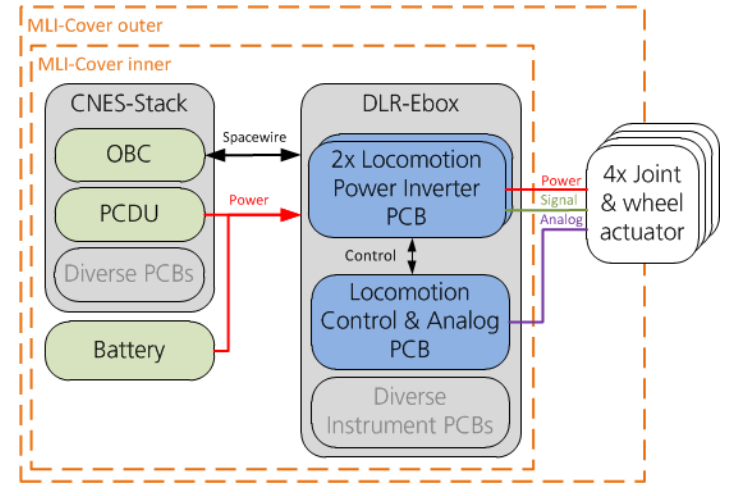

Figure 2. Electronics architecture of CNES and DLR electronics stack

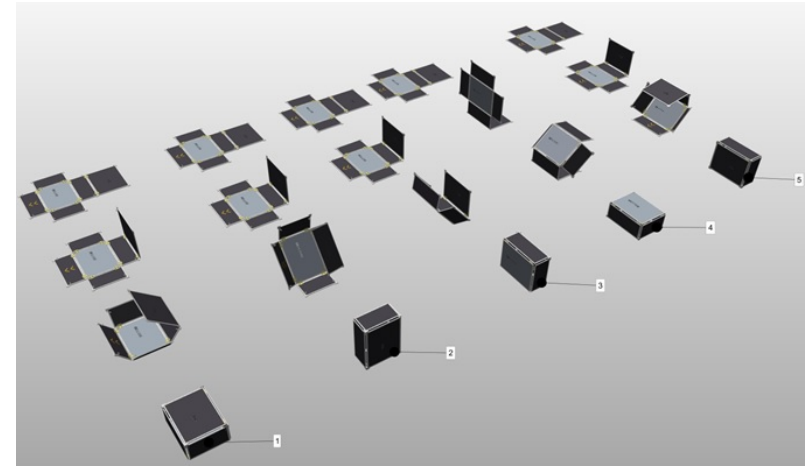

Figure 3. Landing box concept that uprights the rover by unfolding the side walls in a predefined order. Different scenarios are shown: 1. Bottom plate on ground; 2. Side plate right/left on ground; 3. Rear plate on ground; 4. Top plate on ground; 5. Front plate on ground

faces are shown in Figure 2.

The main electronics of the locomotion consist of three PCBs including one control/analogue and two power inverter (PI) boards. The PCBs are connected together via a backplane concept and located in the DLR-Electronic box, which is itself connected via cables to the CNES electronic stack. As the inner compartment offers a more relaxed temperature range than the intermediate space, the main electronic is located inside. In the outer box the actuators are mounted to the side panels, as are the torque and position sensors of the motors and joints. Since these contain some electrical components (e.g. hall sensors), extended thermal, thermal cycling tests above the rated operating and storage temperature ranges are necessary. Via shielded cables, fed through the thermal insulation of the inner box, the main electronics and the motors/sensors are connected.

Apart from the local core voltage supply of the FPGA and supply for the joint position sensors, the needed power supply lines e.g. digital bank supply are provided by the CNES power conditioning and distribution unit (PCDU). To drive the motors the PIs use the non regulated bus which is directly connected to the rover's battery pack.

The control PCB consists of the FPGA with SpaceWire communication interface and its core power supply. It is supervised by a reset and watchdog controller, which observes the core / bank power supply lower levels. An analogue electrical interface for the joint position (JPS) and joint torque sensors (JTRQ) is also located on the PCB. This interface consists of differential amplifiers and impedance conversion for the potentiometer of the JPS. The conditioned and amplified analogue signals are digitised by the on-board analogue-todigital converter (ADC). The analogue signals of both sensors are directly connected to the board via shielded cables. The control signals for the PIs are connected via the backplane connector.

Both PI-PCBs are of the same design. One offers the functionality to drive four 3-phase brushless direct current (BLDC) motors. One PCB is connected to four joint actuators, and the second to four wheel actuators. The motion controller design is a further development from the MASCOT project and the prototype presented in [7]. To decrease electromagnetic interference (EMI) on the non regulated bus, caused by switching the inverters, a power line second order filter is mounted on the board. The filter is built out of an inductance and intermediate circuit capacitors located on each of the boards. Each of the four integrated PI stages locates a bottom side current measurement which gives the current combined over the three half H-bridges. The current is measured with the shunt principle in positive and negative direction. It is then conditioned and digitised with on-board operational amplifiers and ADCs. As the power budget for the rover is limited due to battery and solar panel size and weight, the board can switch off the supply for the PI stages and the motor commutation sensors per board. For the scenario, the rover is driven without using its joint actuators. As such, one PIBoard can be held in power saving state.

\section{Mechanical design}

The mechanical design has three main drivers. First, is to develop a mechanical structure able to survive the impact of the system during the landing phase. Second is to cope with the thermal constraints given by the Phobos surface environment. Third, the design has to respect mass as well as size limitations. In particular, the volume within the rover body is limited to accommodate scientific payloads and internal infrastructure. The low gravity on Phobos does not require powerful drive units, but as there is no knowledge about the soil conditions, the drives still need to overcome adhesion, friction and cold welding issues.

As decribed previously, the rover needs to upright on its own into nominal orientation after landing. In [8], the option of using crushable shells for ballistic landing is investigated. Additionally, mechanisms have been studied to help the lander to survive and achieve a nominal orientation by default, as is depicted in Figure 3. However, the lander is too small to afford mass budget on a supplementary structure. This, in conjunction with the fact that some scientific instruments may need a certain distance to the soil for investigation, demand the concept of using legs to accomplish both. During launch and impact the rover will be prone to vibrations and unknown peak loads. Having a relatively high mass (wheel) at the end of the lever arm (leg) thus requires additional locking mechanisms for the legs. The locking mechanism comprises of an hold down and release mechanism located on the axis of the wheel, which will engage the central part of the wheel and three additional mechanisms located on the circumference of the wheel hub. These three additional mechanisms will support the outer wheel hub during impacts and decrease vi- 


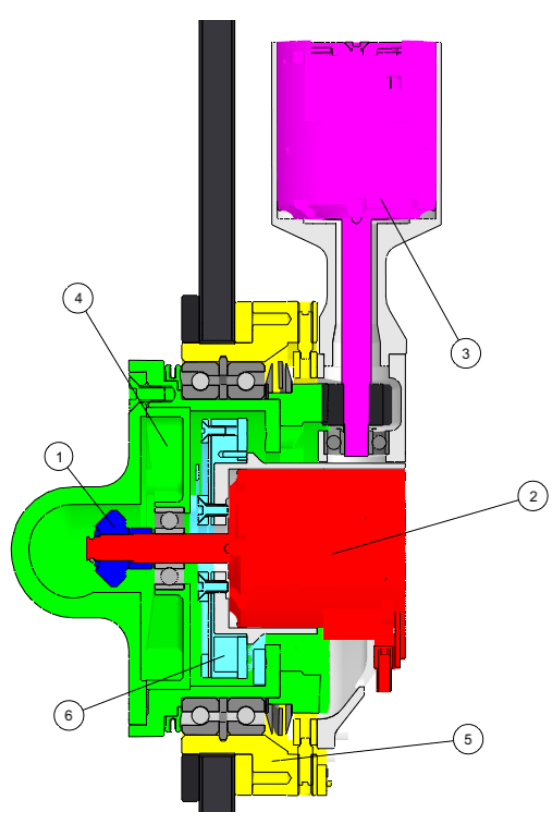

Figure 4. Cross section view of the wheel and shoulder drive unit: 1 . bevel gear, 2. wheel actuator, 3. shoulder actuator, 4. leg structure, 5. torque sensor, and 6. potentiometer assembly.

brations by creating a closed force flow loop with the central hold down and release mechanism. Furthermore, the legs can only guarantee successful up-righting from four of six sides the rover can land on. Therefore, a supplementary, small mechanism is needed to increase the up-righting success probability to an acceptable margin. The latter two aspects are subjects of current development.

As previously stated, the drive units for both wheel and shoulder joints are located inside of the rover body and not in the wheel hub. The units are set up identically for testing and integration purposes. Figure 4 depicts a sectional view of the shoulder joint. The red area shows the drive train for actuating the wheel, the pink area represents the shoulder actuator that is moving the leg structure (green). Hall elements are used for the commutation of the BLDC motors. The motor rotation is coupled to a planetary gear to increase the output torque in the early stage. With the sun gear being comparably small in diameter, the effective radius of friction forces in the gear teeth can be kept small correspondingly, to avoid stiction caused by solidifying grease at low temperatures. The torque is then fed into a custom Harmonic Drive gear box to further increase the torque and to reduce the rotational speed.

A bevel gear (dark blue) transmits the rotation to the wheel hub via an internally running shaft. At the hub, the shaft rotation is transformed to the wheel rotation by using a crown gear. The crown gearing allows for axial displacements of the internal shaft that might be encountered due to different temperature-induced elongations. Consequently, the overall transmission ratio is $1: 2227$. The high ratio provides a uniform, yet extremely low travelling velocity that significantly the escape velocity of Phobos, while maintaining an adequate motor rotational speed of more than $300 \mathrm{rpm}$ for the motor commutation. In contrast to the wheel actuation, the shoulder joint does not require the bevel gear and internal shaft. Apart from this difference, the mechanical transmissions are the

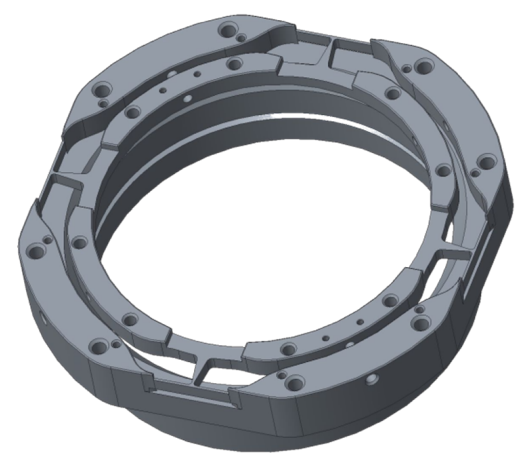

Figure 5. CAD drawing of the torque sensor

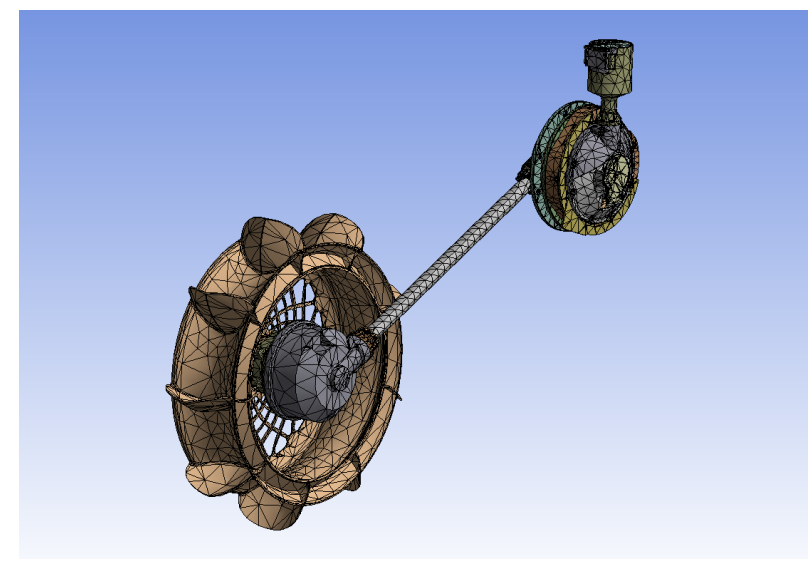

Figure 6. Rover wheel and leg with the drive unit for shoulder joint and wheel hub

same, and allows for infinite rotations of the shoulder joint. The light blue area shows the location of the potentiometers used to measure the absolute position of the rover shoulder joint. As this is critical sensor information, two potentiometers are used with two different sensor principles in order to achieve dissimilar redundancy.

In addition, a torque sensor (yellow) is constructed as spoked wheels. Each wheel is equipped with a strain gauge fullbridge and a temperature sensor. The measuring range is in the magnitude of $2 \mathrm{Nm}$ at a resolution of 12 bit. For overload conditions, a maximum torque of $7 \mathrm{Nm}$ was considered. A CAD drawing of the shoulder joint is shown in Figure 5. To prevent the spokes from torques greater than $7 \mathrm{Nm}$ the sensor provides radial overload bolts. Measuring the rover weight and the driving loads is a challenging task due to the extreme low gravity. During the commissioning phase, the rover automatically starts to extend its locomotion subsystem to the final configuration. The measured torque serves as valuable information to perceive wheel contact with the ground while driving, as well as detection of an obstacle or a stuck leg.Finally, the concept for a generic rover leg is shown in Figure 6.

\section{Wheel-Design}

As the demanding traction conditions of Phobos together with the mass and landing requirements, the MMX wheel design has been optimised in a multi-disciplinary fashion. The core 


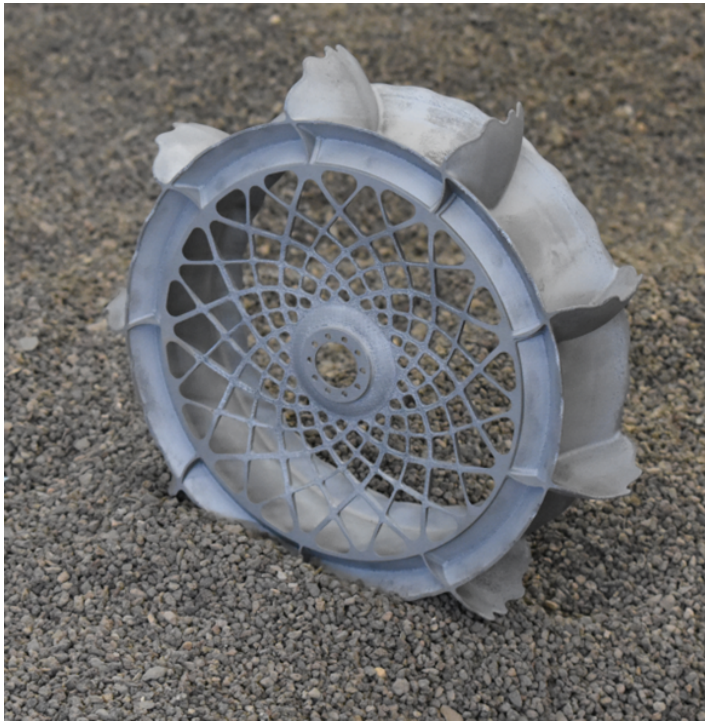

Figure 7. The MMX rover wheel design (breadboard)

design of the traction rim is completed based on the DLR particle simulation tool partsival [9] using DEM and preliminary wheel optimization studies $[10,11]$. Figure 7 shows the breadboard (BB) wheel design, where forward direction of travel rotates counter-clockwise. The traction shape is asymmetric, as driving backwards is a contingency case of operations. The point turn capability shall be available, and will be a scenario in upcoming optimisation campaigns for the later wheel versions. The grouser shape as well as the rim shape are based on the experience in [10] and [11]. The ground regolith material is transported behind the wheel, leaving shallow tracks to be multi passed and thereby using the pushed material totally to create traction. As less material is pushed to the wheel sides, the wheel trail are of low compression and may be easily cut by the wheel during skid-steering. Furthermore, the shape lowers the amount of bulldozing significantly, thus also lowering the resistive force while increasing the tractive efficiency. For further information on the effects of the wheel shape, please refer to [10] and [11]. The strength and mass optimisation are bound together and based on iterative FEM simulations using Ansys and its topology optimisation capabilities. The key to finding optimal compromises between traction and structural strength is to use traction features for structural support, and vice versa. As an example for this type of function integration, the side grousers as well as the main grousers are used as interface for the launch locks, as they pose the highest strength points in the wheel rim and do not have considerable discontinuities in stiffness. Using this approach, the wheel mass is as low as $150 \mathrm{~g}$. As the material of choice, high strength aluminum with hardening effects in cold temperatures is chosen. This allows for uniform material and thermal behaviour at the interfaces to the locomotion system. Furthermore, aluminum features sufficient conduction of electric current in order to suppress the sticking and clogging of soil due to electrostatics, which could harm the traction behaviour massively. Manufacturing of the wheel is carried out by milling to avoid shrink and blow holes, which can occur with casting or laser sintering.

\section{Locomotion Control Software (LCS)}

Locomotion control is a partition on the $\mathrm{OBC}$ that expects motion commands from command control, which is another

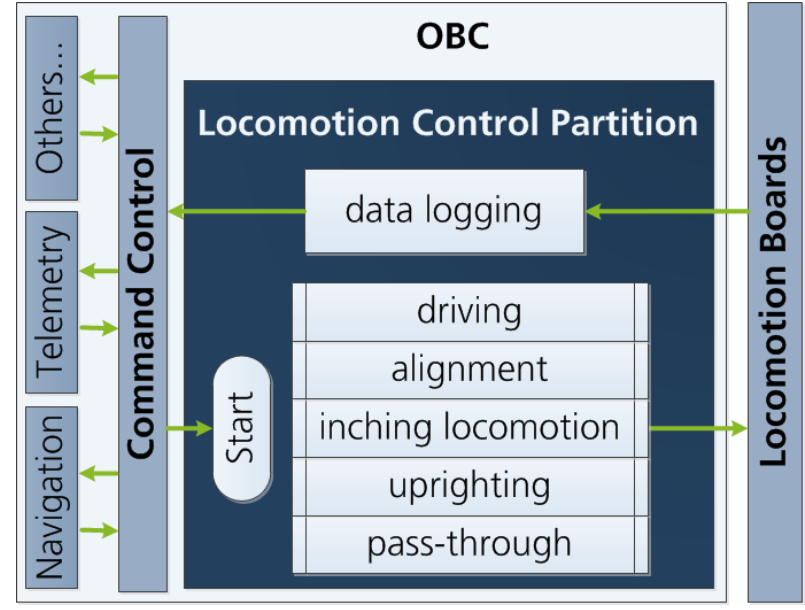

Figure 8. The role and functions of locomotion control in the overall rover software architecture

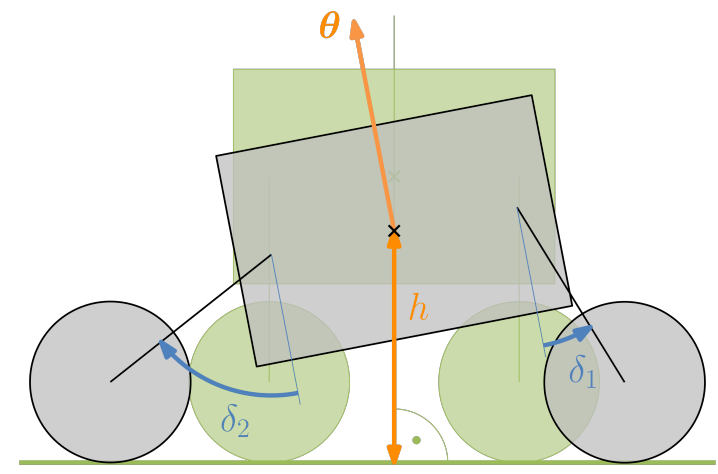

Figure 9. Illustration of the MMX reference plane in green, versus an actual rover position in gray

partition arranging commands and data exchange of all the other rover parts, as shown in Figure 8. From these motion commands, the LCS calculates how the eight wheel and shoulder motors have to be actuated so that the rover fulfills the desired movement. The outcome of these calculations, which is then sent to the locomotion boards (cf. Section 3), is computed in different locomotion modes:

- The driving mode receives the desired rover forward velocity and angular velocity around the yaw axis, and, as an option, a desired body height. The wheel motor commands are calculated by a skid-steering algorithm. To solve the over-actuation of the rover, geometric constraints are used by defining an instantaneous center of rotation.

- The alignment mode receives a desired body orientation and the average distance to a reference plane, see Figure 9. This reference plane is assumed to be the flat, rigid plane underneath the rover if it would be in its maximal height position. The shoulder angles to achieve the desired configuration are calculated through the rover kinematics and the wheel rates are computed for rolling with no slip due to assumed flat terrain.

- The inching locomotion mode [12] is a special mode for the rover to crawl by alternately moving the front and the rear wheels. According to the authors, inching produces approximately $100 \%$ more drawbar pull than rolling. This 
mode thus enables a much higher traction performance in soft sands or steep slopes, as compared to standard driving, which leads to lower slip and increased safety.

- For the uprighting mode, the first action after landing, an uprighting sequence for the rover will be provided by CNES. Here, different limit requirements are checked, as compared to all the other modes, as the solar panels are not expanded yet and therefore, the legs can perform a $360^{\circ}$ turn only during this phase.

- For special motions where the motor rates are commanded directly, there is a passing-through mode which only checks if the desired values are valid.

In addition to its main purpose, the LCS will perform further tasks. Before the locomotion system can act, a startup procedure has to be performed. The locomotion boards (see Section 3) are checked for their operation readiness, in particular, for the need to be heated up to the required temperature range. This will be done first at the beginning of an operation day then regularly while the LCS is running. Moreover, the LCS will collect the log and housekeeping data from the locomotion boards, and send these data packages further to command control. Figure 8 summarizes the LCS tasks and interactions with other software components.

\section{FPGA Firmware and Communication}

On the control PCB, a FPGA is used to implement the communication interface towards the $\mathrm{OBC}$, the current controllers for all motors and interfaces to sensors, as well as other peripherals. The high parallelism of FPGAs, and the high number of IO-Ports allow the integration of all those features on one single chip.

Robust communication between control $\mathrm{PCB}$ and $\mathrm{OBC}$ is critical to locomotion since communication errors can result in physical damage to the rover. Therefore, the communication between control PCB and OBC follows a handshake scheme. A read or write request is sent from the OBC to the control PCB and answered with a reply. The reply contains the requested data (in case of a read access) and a status field to indicate a successful memory access or to transmit an error code. The SpaceWire (SpW) [13] Remote Memory Access Protocol (RMAP) [14] is used to implement this communication scheme. It allows packet oriented communication and $\mathrm{CRC}$ protection, which enables the detection of communication errors like bit flips or incomplete packages. Additionally, SpW RMAP can seamlessly be connected to an On-Chip Bus for communication. A SpW RMAP target is implemented on the control PCB's FPGA. Data registers of all modules inside the FPGA are connected via an on-chip bus to the SpW RMAP target.

These data registers are used for configuration, control, and housekeeping. For each of the rover's eight motors, an instance of the same motor module is used for commutation and functional safety features. Each motor module has its own set of registers to receive commands and provide sensor and status information. Additionally, there are data registers representing the sensor values of each ADC. The sensor data is read periodically, and written into the corresponding data registers.

\section{Modeling and simulation}

The environmental properties of Phobos are currently unknown at a resolution and fidelity to a scale usable for rover locomotion. It is nonetheless to be expected to be drastically different from the Earth's environment. This makes physical experiments to determine system behaviour difficult to impossible, thus making simulation the only viable option for testing and analysis. These simulations, visualized with the DLR Visualization Library [15] in Figure 10, are based on the Rover Simulation Toolkit [16] that builds upon the Dymola implementation with the Modelica modeling language. The core model build includes multi-body dynamics, drive train mechancis, self and external collisions, as well as terramechanical models for ground contact simulations. [17,18]. The process of the modelling and simulation activities is to build, refine and utilize the models constantly during all mission phases.

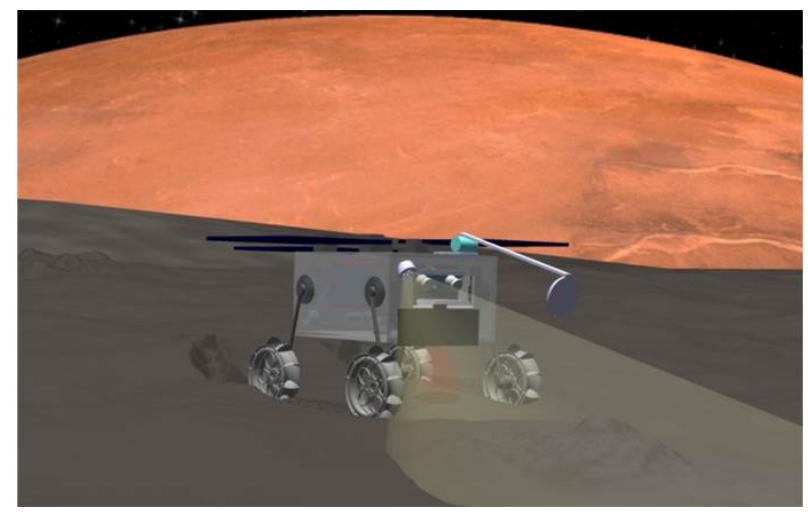

Figure 10. Simulation of the MMX rover visualized by the DLR SimVis tool

\section{Landing impact simulation}

As an example of the simulation capability, a closer examination to the landing of the rover on Phobos is taken. As of writing of this paper, the rover is planned to be separated from the MMX spacecraft at a height of $50 \mathrm{~m}$. After separation, the rover will descend without any deceleration and impact the ground at a velocity of $\approx 0.75 \mathrm{~m} / \mathrm{s}$. The shock loads during this initial impact are another design driver for parts of the locomotion system. An understanding of the load magnitude, profile and distribution between each component is critical during component design and experiment design. To predict these loads, the model has to correctly depict the mechanical structure with its dominating flexibilities in the wheels, hold down and release mechanisms (HDRMS), as well as the chassis.

Due to the high uncertainty regarding surface geometry and parameters, a worst case condition must be assumed. This would be an impact on a rock that is either buried or has a sufficient mass to be considered as fixed. With an impact velocity of $\approx 0.75 \mathrm{~m} / \mathrm{s}$ and a very short time scale of $<0.5 \mathrm{~s}$, effects of gravity can be neglected. Based on these simplifications an impact with varying directions on a flat surface is a representative scenario to analyse the impact. Contact parameters of the rover components are based on their physical properties. The environment is set to depict a hard stone such as granit. One simulation run provides the resulting forces acting on each component for a specific impact direction. Figure 11 shows the impulse experienced at the wheel hub for different impact orientations. This reveals the critical cases that need analytical in simulation or physical testing in more detail. 


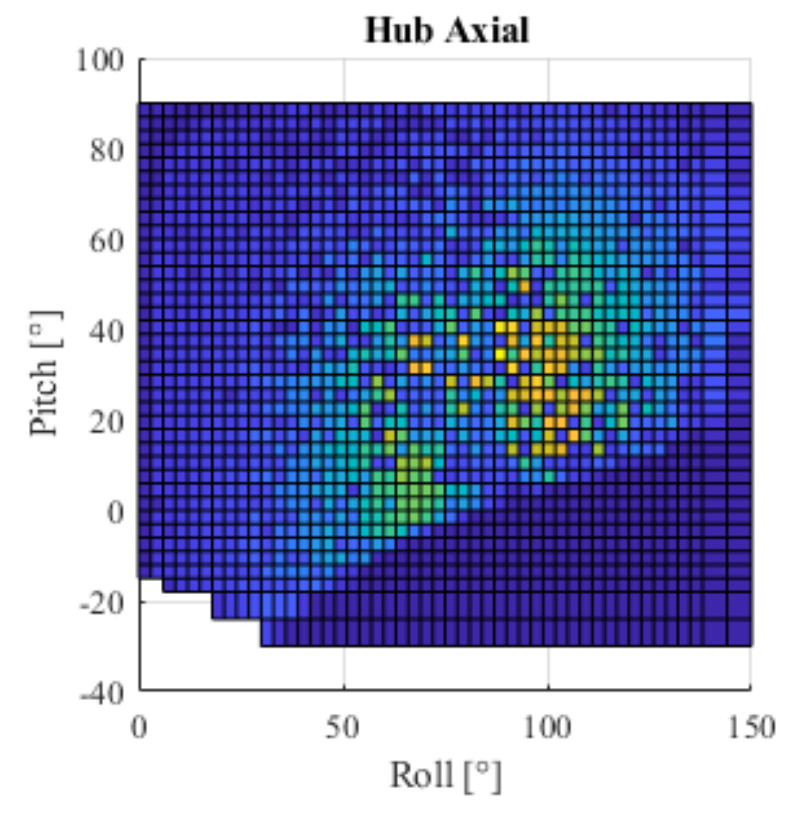

Figure 11. Landing impact impulse in axial direction on the wheel hub for different rover attitudes

\section{Analysis AND TeSting}

\section{Thermal Analysis}

The thermal control and behaviour of the MMX locomotion system is analysed and optimized in a Finite Element model (FEM) in parallel with the mechanical and electronics development. Since the drive train (see Figure 6) is a highly integrated multi-body system, in addition to typical simplification approaches, the main assembly model is further divided into 14 different thermal sections. Each section is defined as a group of linked bodies, which, in contrast to the intersections, have a comparatively high thermal contact conductance. The section borders include point contacts (e.g. ball bearings), line contacts (e.g. gears), clearance fits and heat transfer between bodies solely through thermal radiation. This measure allows a rapid setup and rearrangement of thermal models, considering different complexities that are vital for the respective analysed cases. In addition, the analysis benefit from the improved efficiency in terms of convergence time and data space. For the model parametrization, thermal vacuum chamber tests on component and section level will be conducted and subsequently correlated.

\section{Radiation Test}

A Texas Instruments DRV8332 automotive rated brushless DC motor controller has been selected for its size and form factor for the power inverter board. This commercial off the shelf (COTS) part offers extended storage temperature range of $-55^{\circ} \mathrm{C}$ up to $+150^{\circ} \mathrm{C}$ and the operating temperature range of $-40^{\circ} \mathrm{C}$ up to $+125^{\circ} \mathrm{C}$. These specifications are close to the space grade temperature range, which minimize the effort of the up-screening process.

To get a full picture of the radiation performance, a total ionizing dose (TID) test, a proton test, and a heavy ion test were conducted. The TID and the proton test were performed at the Helmholtz Zentrum Berlin Wannsee (HZB, [19])) with biased samples. For the TID test, a dose rate of 3.82
$\mathrm{Gy}(\mathrm{Si}) /$ hour up to $550 \mathrm{~Gy}(\mathrm{Si})$ was applied. For the proton test, a fluence of $1.7 \mathrm{E}+10$ protons per square centimetre was applied. Three different energy levels were used for this campaign: $30 \mathrm{MeV}, 50 \mathrm{MeV}$ and $68 \mathrm{MeV}$ per proton. During the irradiation some important parameters were monitored in-situ with specially designed test hardware. Finally, a heavy ion test was performed at the radiation effects facility in Jyvaskyla (RADEF, [20]), where Nitrogen, Krypton and Xenon ions were used. The applied fluence was $1.0 \mathrm{E}+10$ ions per square centimetre for each ion species.

For up to $550 \mathrm{~Gy}(\mathrm{Si})$, all measured parameters stayed within their rated limits. The latch-up threshold LET of the device could be found at $59.9 \mathrm{MeV} \cdot \mathrm{cm} 2 / \mathrm{mg}$. All these test results indicate the DRV8332 to be sufficient for usage within this mission. For more details, please refer to [21].

\section{Thermal Tests}

To ensure survivability of the rover in extreme low temperatures, multiple thermal test campaigns will be conducted. One run was performed in order to determine the limits of the operational range of the hall sensors which are treated as flight candidates. Figure 12 shows the recorded temperatures over the test time in seconds. The output signals of the sensors were recorded and analysed over the entire run. In the rated temperature range down to $-40^{\circ} \mathrm{C}$, a small temperature depending deviation of the switching characteristics could be measured. In comparison to the component specification, the switching hysteresis of the sensor is constant, but shifted on the magnetic flux density axis. At below $-60^{\circ} \mathrm{C}$, the sensors autonomously stopped their operations.

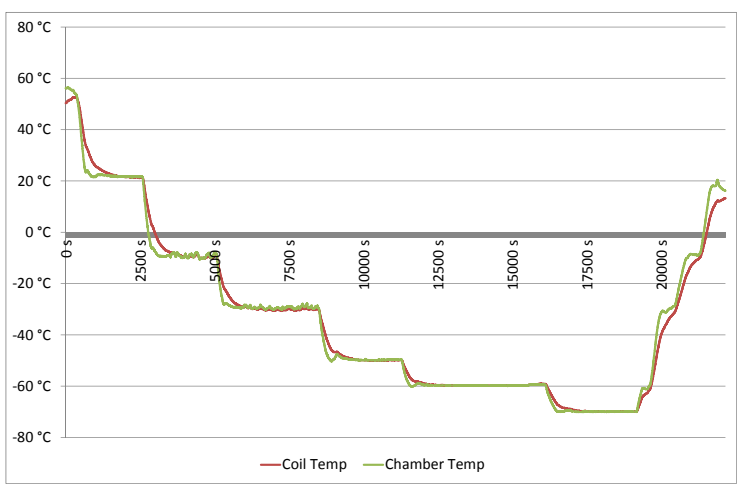

Figure 12. Temperature profile for sensor test

The next test campaign will be a thermal cycling test where the sensors are required to survive multiple transitions between $-130^{\circ} \mathrm{C}$ and $+60^{\circ} \mathrm{C}$. One of the aims of this campaign is the determination of the minimum required temperature in the rover and to get an idea of the mechanical stability of the tested sensors. This huge temperature range and the high number of thermal cycles lead to a serious mechanical stress, which may lead to a mechanical destruction of the sensor, based on demolishing of the internal bond wires, for example. Finally, the quality of the conducted thermal tests are the key to success for this mission. 


\section{Conclusion}

This paper presented on-going development of the MMX rover targeted for Phobos, with the focus on the locomotion subsystem. The harsh environmental conditions and the high demand for robust and reliable operation present many challenges to the locomotion subsystem. Starting with a brief mission description, the paper discussed the developed concept to ensure actuation of the rover. This demanding area of robotics-driven space exploration is of increasing interest to the space community, the close cooperation of JAXA, CNES and DLR is critical to its success.

\section{ACKNOWLEDGeMent}

The authors of this paper would like to extend their thanks to their colleagues at the Robotics and Mechatronics Center at DLR for their tireless support: Manuel Maria Bras Pereira, Rainer Krenn, Manfred Schedl, Bernhard Vordermayer, and Tim Wagner.

\section{REFERENCES}

[1] J. Reill, H.-J. Sedlmayr, P. Neugebauer, M. Maier, E. Krämer, and E. Lichtenheldt, "MASCOT - Asteroid Lander with innovative Mobility Mechanism," in 13th Symposium on Advanced Space Technologies in Robotics and Automation (ASTRA), ESA/ESTEC, Noordwijk, Netherlands, May 2015.

[2] T.-H. Ho, V. Baturkin, R. Findlay, C. Grimm, J.-T. Grundmann, C. Hobbie, E. Ksenik, C. Lange, K. Sasaki, M. Schlotterer, M. Talapina, N. Termtanasombat, E. Wejmo, L. Witte, M. Wrasmann, G. Wübbels, C. Rößler, J.and Ziach, J. Biele, C. Krause, S. Ulamec, M. Lange, O. Mierheim, J. Lichtenheldt, M. Maier, J. Reill, H.J. Sedlmayr, P. Bousquet, A. Bellion, O. Bompis, C. Cenac-Morthe, M. Deleuze, S. Fredon, E. Jurado, E. Canalias, R. Jaumann, J.-P. Bibring, K. H. Glaßmeier, M. Grott, L. Celotti, F. Cordero, J. Hendrikse, and T. Okada, "MASCOT - The Mobile Asteroid Surface Scout onboard the HAYABUSA2 Mission," in Space Science Reviews, Volume 1 / 1962 - Volume 199 / 2016, Springer, April 2016.

[3] Fujimoto et al., "JAXAs Martian Moons eXploration," in European Planetary Science Congress, 2017.

[4] Tardivel, Lange et al., "The MMX rover: An innovative design enabling Phobos in-situ exploration," in Proceedings of LCPM, 2019.

[5] Ulamec et al., "A Rover for the JAXA MMX mission to Phobos," in 70th International Astronautical Congress, 2019.

[6] Bertrand et al., "Roving on Phobos, Challenges of the MMX rover for space robotics," in Proceedings of ASTRA, 2019.

[7] M. Maier, M. Chalon, J. Reill, and H.-J. Sedlmayr, "Highly integrated, radiation-hardened, motor controller with phase current measurement," in 14th Symposium on Advanced Space Technologies in Robotics and Automation (ASTRA), ESA/ESTEC, Noordwijk, Netherlands, June 2017.

[8] S. Schröder, C. Grimm, and L. Witte, "A crushable shell for small body landers," in DLRK 2018, September 2018, https://elib.dlr.de/121939/.
[9] R. Lichtenheldt, S. Kerler, A. Angerer, and W. Reif, "partsival - collision-based particle and many-body simulations on gpus for planetary exploration systems," in The 5th Joint International Conference on Multibody System Dynamics, June 24 - 28, Lisboa, Portugal, 2018.

[10] S. Ono, R. Lichtenheldt, K. Nagaoka, J. Bals, and K. Yoshida, "Performance analysis of wheel for mars rover by using discrete element method simulations," Journal of Terramechanics, 2019 (submitted).

[11] R. Lichtenheldt, "Lokomotorische Interaktion Planetarer Explorationssysteme mit weichen Sandböden Modellbildung und Simulation," Ph.D. dissertation, Ilmenau University of Technology, ISBN 978-3-84392704-8, 2016.

[12] S. Moreland, K. Skonieczny, D. Wettergreen, V. Asnani, C. Creager, and H. Oravec, "Inching locomotion for planetary rover mobility," in 2011 Aerospace Conference, March 2011, pp. 1-6.

[13] European Cooperation for Space Standardization (ECSS), "ECSS-E-ST-50-12C Rev.1 - SpaceWire - links, nodes, routers and networks," 2019, http://ecss.nl/standard/ecss-e-st-50-12c-spacewirelinks-nodes-routers-and-networks/.

[14] European Cooperation for Space Standardization (ECSS), "ECSS-E-ST-50-52C - SpaceWire - remote memory access protocol," 2010, https://ecss.nl/standard/ecss-e-st-50-52c-spacewireremote-memory-access-protocol-5-february-2010/.

[15] M. Hellerer, T. Bellmann, and F. Schlegel, "The DLR Visualization Library - recent development and applications," in Proceedings of the 10 th International Modelica Conference; March 10-12; 2014; Lund; Sweden, no. 96. Linköping University Electronic Press, 2014, pp. 899-911.

[16] M. Hellerer, S. Barthelmes, and F. Buse, "The DLR Rover Simulation Toolkit," in 14th Symposium on Advanced Space Technologies in Robotics and Automation (ASTRA), 20-22 June 2017, Leiden, The Netherlands, 2017.

[17] R. Lichtenheldt, S. Barthelmes, F. Buse, and M. Hellerer, "Wheel-ground modeling in planetary exploration: From unified simulation frameworks towards heterogeneous, multi-tier wheel ground contact simulation," in Multibody Dynamics - Computational Methods and Application, ser. Computational Methods in Applied Sciences, J. M. Font-Llagunes, Ed. Springer, 2016, vol. 42, ch. 8, pp. 165-192.

[18] F. Buse, "Using superposition of local soil flow fields to improve soil deformation in the DLR soil contact model - SCM," in The 5th Joint International Conference on Multibody System Dynamics, June 24-28, 2018, Lisbon, Portugal, 2018.

[19] Helmholtz Zentrum Berlin Wannsee, "Cobalt60 source," 2017, https://www.helmholtzberlin.de/industrie/techniken/processing_de.html.

[20] University of Jyvaskyla, "Radiation effects facility - RADEF," 2017, https://www.jyu.fi/science/ en/physics/research/infrastructures/acceleratorlaboratory/radiation-effects-facility.

[21] H.-J. Sedlmayr et al., "Radiation test of a BLDC motor driver component," in 2018 IEEE Radiation Effects Data Workshop, 2018. 


\section{BIOGRAPHY}

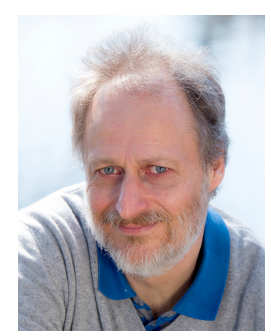

Hans-Juergen Sedlmayr received his Dipl.-Ing degree in Electrical Engineering from the University of Applied Science Munich in 1992. Since 2001, he has been with the German Aerospace Center, Institute of Robotics and Mechatronics. His main research focus is in the field of radiation testing of electric and electronics parts and embedded software development inside robots for terrestrial and space applications.

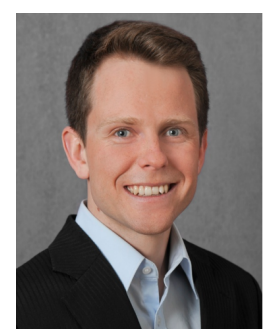

Stefan Barthelmes received his B.Sc. and M.Sc. degree in Mechanical Engineering from Technische Universität München. He currently works as a research associate at the Institute of System Dynamics and Control of the German Aerospace Center (DLR). His main research focus is model-based chassis control and simulation model development of planetary exploration rovers. Within the MMX mission, he is responsible for the control software of the locomotion subsystem.

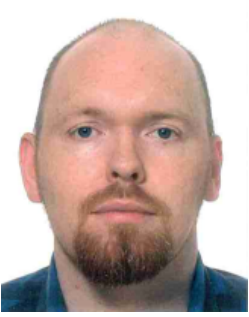

Ralph Bayer received his Master of Science (M.Sc) in Mechanical Engineering from the Regensburg University of Applied Sciences, Germany, in 2011 and joined the German Aerospace Center (DLR) in 2010. He was a member of the development team of the space qualified force feedback joystick used for the KONTUR-2 ISS-toground tele-manipulation experiments in 2015?2016, as well as the METERON SUPVIS Justin ISS-toground telerobotic experiments in 2017-2018. He currently serves as the lead thermal engineer at DLR's Institute of Robotics and Mechatronics. Mr. Bayer plays a key role in the thermal design of the MMX locomotion system.

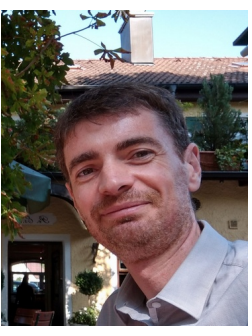

Wieland Bertleff received his Dipl.Ing $(F H)$ in Electrical Engineering degree from the University of Applied Sciences in Heilbronn in 2002. In 2007, he received his M.Sc. degree in Electrical Engineering from the University of Applied Sciences, Munich. After his diploma, he started to work at the German Aerospace Center at the Institute of Robotics and Mechatronics in Oberpfaffenhofen where he is involved in the development and design of force and torque sensors for robotic systems and devices for human machine interfaces. He also contributed in the robotic mission ROKVISS in the field of friction identification and he increasingly focuses on qualification and test campaigns for space hardware, in particular the current MMX mission.

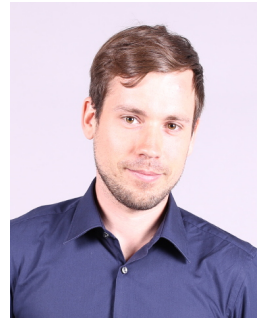

Markus Bihler received his M.Sc. degree in computer science from the University of Applied Science Augsburg, Germany in 2015. Since then, he has been with the German Aerospace Center, Institute of Robotics and Mechatronics. His main research focus is the field of FPGAs, communication and System-onChip architecture inside robots. Since October 2017, he is group leader for digital electronics within the department Mechatronic Systems.

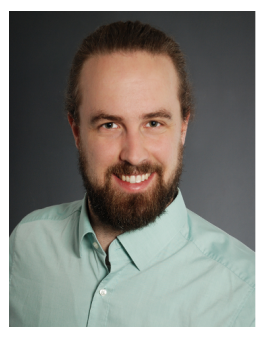

project.
Fabian Buse received the degrees of B.Sc. and M.Sc. from RWTH Aachen University. Since 2015, he has been Research Associate at Institute of System Dynamics and Control. His research interests are in terramechanics for planetary rovers. He is the lead engineer of the DLR Terramechanics Robotic Locomotion Lab (TROLL) and is leading the rover simulation for the MMX rover

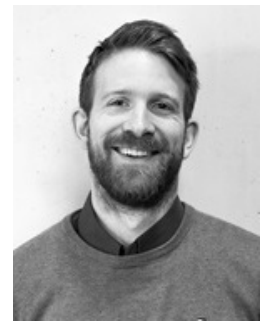

Arm System.
Maxime Chalon received a master degree in mechatronics and a Ph.D from the university of MinesParisTech. in robotics. He is currently leading the development of the locomotion subsystem. Before his current rover activities, he led the development of the Spacehand, a space robotic hand at DLR as well as working as a control engineer on the tendon driven hand of the DLR Hand

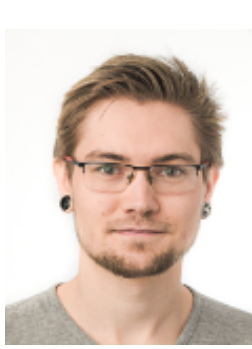

Dennis Franke joined the German Aerospace Center in 2018. He works as technical engineer at the Institute of System Dynamics and Control. Currently, he is working on different aerospace topics. In the MMX rover project, he is the main designer of the wheel.

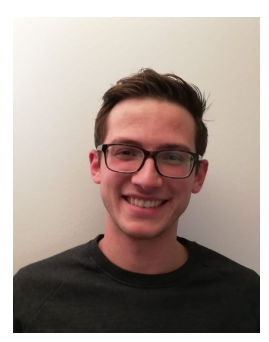

Florian Ginner received his B.Eng. in Mechatronics and Precision Engineering from the Munich University of Applied Sciences and the M.Sc. degree in Mechatronics and Information Technology from the Technical University of Munich. He currently works as a research associate at the Institute of Robotics and Mechatronics of the German Aerospace Center (DLR). His main research interests include the design and analysis of robust actuators for terrestrial and space applications, as well as hybrid wheellegged locomotion concepts. 


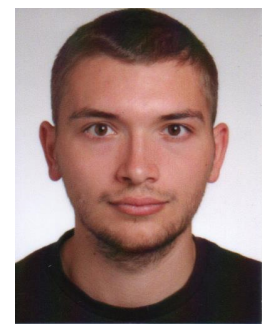

Viktor Langofer received the degrees of B.Sc. and M.Sc. from Technische Universität Kaiserslautern. Since 2017, he has been Research Associate at German Aerospace Center (DLR), Institute of Robotics and Mechatronics. His main area of activities are research in compliant and tendondriven mechanisms in humanoid robotics, as well transmission design for space applications. He is responsible for the mechanical design of the locomotion subsystem of the MMX rover.

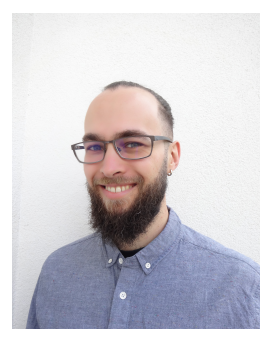

Roy Lichtenheldt received his B.Sc./M.Sc. and Dr.-Ing. degrees in mechatronics from Ilmenau University of Technology. He currently works at the Institute of System Dynamics and Control of DLR and is responsible for particle based terramechanics, the simulation framework partsival. Dr. Lichtenheldt provided in depth analysis and optimization for the $H P^{3}$ Mole for NASA's InSight mission to Mars and developed the trajectory optimization tool for the MASCOT lander. He is also leading the wheel development for the MMX rover and is the system lead for the DLR Scout rover for exploration of extreme terrain and planetary caves.

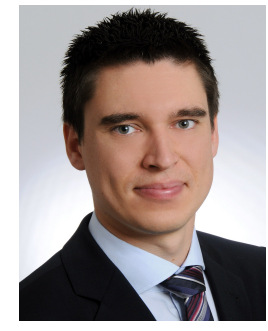

Thomas Obermeier received the degrees of B.Eng. and M.Eng. from the University of Applied Sciences Munich. Since 2014, he works as a research associate at the Institute of Robotics and Mechatronics of the German Aerospace Center. He is mainly working on the design of electronic concepts and its circuitry for robots in space applications. For the MMX mission, he is responsible for the electrical design of the locomotion subsystem.

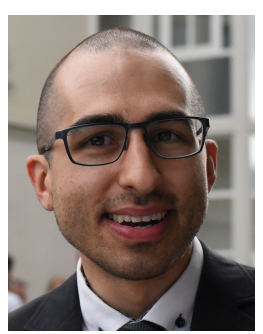

Antoine Pignède received a double degree in electrical engineering and cybernetics from the Technical University of Darmstadt and the Norwegian University of Science and Technology in Trondheim in 2016. Since October 2016, he has been a member of scientific staff of the Institute of System Dynamics and Control at the German Aerospace Center DLR. His research area concerns modelling, simulation and optimization of planetary exploration rovers.

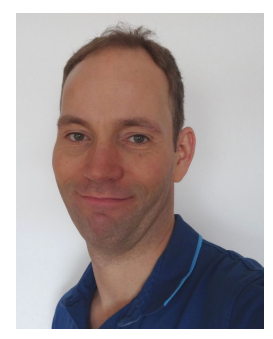

Josef Reill received the degree in electrical engineering from the FriedrichAlexander-Universität Erlangen-Nürnberg, Germany in 2005 and the Dr.-Ing. degree in 2010. Since 2009, he is with the German Aerospace Center, Institute of Robotics and Mechatronics. His main area of activity is the control of permanent magnet synchronous machines used in robotic joints for terrestrial and space applications. He is leading the electrical drives activities at the institute and was coordinating the mobility subsystem of MASCOT.

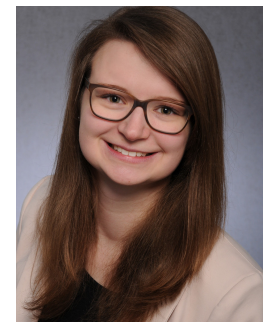

Juliane Skibbe studied Mathematics at the University of Wuerzburg and the Université d'Orléans. She wrote her Master's Thesis at the German Aerospace center on the mathematical modelling of the compliant spokes for a rimless wheel rover. After finishing her Master's degree in 2018, she started working as a research associate at the Institute of System Dynamics and Control of the German Aerospace Center (DLR). Her main focus is on developing locomotion control algorithms for rover for planetary exploration.

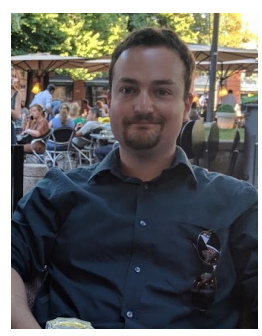

Simon Tardivel received his M.S. in Aerospace Engineering from Supaero (Toulouse, France) in 2010. Under supervision of Dr. Scheeres, he received a Ph.D. in Aerospace Engineering Sciences from the University of Colorado Boulder in 2014, on the topic of the deployment of landers to asteroid surfaces. Since June 2017 he has been working at CNES (Centre National d'Etudes Spatiales) in Toulouse in the future missions design and analysis group, and on the science and engineering of the MMX rover. 